\title{
Capacidades e Dificuldades Socioemocionais de Crianças Antes e Após a Participação no Método FRIENDS
}

\author{
Strengths and Difficulties Social-Emotional of Children Before and After Taking Part of \\ FRIENDS Method
}

\section{Capacidades y Dificultades Socioemocionales de Niños Antes y Después de la Participación en Método FRIENDS}

\author{
Cynthia Lais Ignachewski ${ }^{1}$ \\ Ana Priscila Batista \\ Caroline Guisantes de Salvo Toni \\ Giulia Tatiana Tkaczyk Pavoski \\ Universidade Estadual do Centro-Oeste
}

\begin{abstract}
Resumo
Atualmente, milhões de pessoas sofrem de algum transtorno mental, sendo as crianças uma considerável parcela dessa população. Nesse sentido, intervenções precoces para prevenir e intervir sobre distúrbios na infância são muito importantes. Uma possibilidade de intervenção precoce é o Método FRIENDS, que busca desenvolver habilidades socioemocionais e resiliência. O presente estudo buscou investigar se o Método FRIENDS promoveu melhorias em comportamentos relacionados a capacidades e dificuldades socioemocionais de crianças, relacionadas a comportamento pró-social, hiperatividade, sintomas emocionais, problemas de conduta e de relacionamento com os colegas. Participaram do estudo dez crianças de uma cidade do interior do Paraná com idade entre 6 e 7 anos. Os pais responderam o Strengths and Difficulties Questionnaire (versão em português), antes e depois do método e dois meses após sua finalização. O resultado indicou uma diminuição nas dificuldades dos participantes, demonstrando que o FRIENDS foi eficaz para a melhoria dos comportamentos das crianças.

Palavras-chave: infância, relações interpessoais, habilidades sociais, psicologia preventiva, Método FRIENDS
\end{abstract}

\begin{abstract}
Nowadays, milions of people suffer from some mental disorder, being the children a considerable part of this population. In this sense, early interventions to prevent and intervene on childhood disorders are very important. One possibility for early intervention is the FRIENDS Method, which seeks to develop social-emotional skills and resilience. This work sought to investigate whether the FRIENDS Program brought improvements in behaviors related to children's Strengths and Difficulties socialemotional, related to prosocial behavior, hyperactivity, emotional symptoms, conduct problems and peer relationships. Ten children from a city in the interior of Paraná, aged between 6 and 7 years, participated in the study. The parents answered the Strengths and Difficulties Questionnaire (Portuguese version), before and after the program e two months after its end. The result indicated a decrease in the difficulties of the participants, demonstrating that FRIENDS was effective in improving children's behavior. Keywords: childhood, interpersonal relationships, social skills, preventive psychology,

\section{FRIENDS Methods}

\section{Resumen}

Actualmente, millones de personas sufren algún trastorno mental, siendo los niños considerable parte de esa población. En ese sentido, intervenciones precoces para prevenir e intervenir sobre los disturbios en la infancia son importantes. Una posibilidad de intervención precoz es el Método FRIENDS que busca desarrollar habilidades socioemocionales y resiliencia. El presente estudio buscó investigar si el método FRIENDS promovió mejorías en conductas de capacidades y dificultades socioemocionales de niños, relacionadas a conductas pró-sociales, hiperactividad, síntomas emocionales, problemas de conducta y de relacionamiento con colegas. En el estudio participaron diez niños del interior de Paraná con edades entre seis y siete años. Los padres respondieron el Strengths and Difficulties Questionnaire (versión en portugués), antes y después del método y dos meses

\footnotetext{
${ }^{1}$ Endereço de contato: Rua Coronel Pires, n. 45, Irati, PR, CEP: 84500-000. Tel.: (42) 99905-5283. E-mail: cyn_lais@windowslive.com
} 
después de su finalización. El resultado indicó una disminución en las dificultades de los participantes, demostrando que el FRIENDS fue eficaz para la mejoría de los comportamientos de los niños.

Palabras clave: infância, relaciones interpersonales, habilidades sociales, psicologia preventiva, Método FRIENDS

\section{Introdução}

A saúde mental, segundo Abreu, Barletta e Murta (2015), é um tema que vem ocupando bastante espaço nas discussões acerca do cuidado com a saúde, pois o seu impacto na sociedade está cada vez mais evidente. As autoras mencionam que atualmente mais de 400 milhões de pessoas no mundo sofrem de algum transtorno mental e que, no Brasil, um levantamento epidemiológico, realizado em 2003, indicou que cerca de 20 milhões de brasileiros sofrem com algum tipo de doença mental. Dados tanto nacionais quanto internacionais (Barros, 2005; Fleitlich-Bil, \& Goodman, 2004; Kösters et al., 2012; Stallard et al., 2014) indicam que uma considerável parte dessa porcentagem está relacionada a crianças em idade escolar, índice suscetível a ser muito maior do que os valores das pesquisas indicam, pois, de acordo com Liddle e Macmilan (2010), muitos casos não são diagnosticados.

Problemas relacionados à saúde mental na infância são bastante preocupantes, pois, como assinalam Kösters et al. (2012), tais problemas associam-se a limitações no funcionamento atual das crianças, tais como o envolvimento social, desempenho na escola e baixa autoestima, além de poder afetar negativamente o desenvolvimento social e emocional a longo prazo, sinalizando uma possível psicopatologia na idade adulta. Dentre as consequências futuras acarretadas pelos problemas mentais infantis, Miller et al. (2011) apontam transtornos do humor, aumento de transtornos por uso de substância, maiores taxas do hábito de fumar e tendências suicidas.

Quando se pensa em comprometimentos à saúde mental infantil engloba-se uma ampla gama de dificuldades que podem ser apresentadas pelas crianças, dentre as quais se pode citar: sintomas de hiperatividade, que envolvem inquietação constante, impulsividade, desatenção e distração; sintomas emocionais que se relacionam a ansiedades, preocupações excessivas, medos e características depressivas, como, por exemplo, sensação de inutilidade e desesperança; problemas de comportamento que podem tanto estar ligados a irritabilidade, temperamento explosivo e agressividade quanto a comportamentos antissociais como roubo, mentiras e abuso de substâncias; e, por fim, problemas de relacionamento de ordem social, que envolvem dificuldades nas relações com a maioria dos parceiros sociais, sejam crianças ou adultos, desconhecidos ou familiares, dificultando o apego e desenvolvimento de amizades, podendo provocar o isolamento (Fraga, 2015; Goodman \& Scott, 2012; Liddle \& Macmillan, 2010; Muszkat, Miranda, \& Rizutti, 2011; Stallard, 2010; Stallard, Simpson, Anderson, \& Goddard, 2008).

De acordo com Saud e Tonelotto (2005), a investigação de tais dificuldades é bastante trabalhosa, principalmente dada a complexidade relativa ao tema. Nesse sentido, um instrumento bastante eficaz no levantamento desses dados refere-se ao Strengths Difficulties Questionnaire - SDQ (Goodman, 1997). Além das dificuldades anteriormente citadas, o instrumento também contempla a avaliação de um fator de proteção à saúde mental na infância, relacionado ao comportamento pró-social, isto é, ações benéficas que se pretende 
ofertar aos outros como, por exemplo, ajudar, doar, cooperar e compartilhar, de forma autoindicada ou requisitada (Martin-Raugh, Kell, \& Motowidlo, 2016).

A detecção de dificuldades que se relacionam a fatores de risco à infância e das capacidades que estão ligadas a fatores de proteção são, de acordo com Maia e Williams (2005), crucialmente importantes para atuar preventivamente frente aos problemas de comportamento das crianças. Segundo Murta, Günther e Guzzo (2015), intervenções precoces e promotoras de saúde mental devem ter o maior foco na infância, para tornar possível o fortalecimento diante de circunstâncias nem sempre favoráveis, visto que essa é uma fase privilegiada para a implementação precoce de medidas que reduzam o impacto de risco à saúde e desenvolvam sua promoção.

Em vista disso, percebe-se que intervenções precoces para prevenir e intervir sobre distúrbios na infância são muito importantes. No entanto esse acesso a serviços terapêuticos adequados não está corriqueiramente disponível, pois, como destaca Stallard (2010), poucas crianças recebem qualquer forma de intervenção de um especialista em saúde mental.

Há diversos modos de se realizarem intervenções, dentre esses, Abreu et al. (2015), tendo como base o National Research Council (US) e Institute of Medicine Committee on the Prevention of Mental Disorders and Substance Abuse Among Children, Youth, and Young Adults (CPMDSA), caracterizam três: universal, selecionada e indicada. A primeira é relacionada a atender toda a população sem nenhum risco individual aparente, a segunda se refere à intervenções destinadas a populações que apresentam um risco maior que a média de desenvolver algum transtorno mental, e a terceira inclui estratégias direcionadas a indivíduos identificados com vulnerabilidade aumentada para desenvolver um transtorno mental e/ ou que apresentam sinais ou sintomas subclínicos de transtornos mentais. De acordo com as autoras, considera-se interessante o trabalho com o primeiro modo de intervenção, por acreditar que ele apresenta como vantagens um custo baixo por indivíduo atendido, por ser a intervenção efetiva e aceitável para a população e ter baixo ou nenhum risco (efeito colateral) como consequência negativa da intervenção em si.

O desenvolvimento de competências sociais e emocionais nas crianças é um fator de proteção, pois, como caracterizam Pahl e Barrett (2007), tais competências evitam o surgimento de problemas de saúde mental, o desajustamento escolar, o baixo desempenho acadêmico e ajudam na promoção de sucesso profissional, além de serem importantes também para o desenvolvimento biopsicossocial e na formação dos sujeitos sociais e políticos. Stallard (2010) complementa que essas capacidades também trazem benefícios no bem-estar geral da criança, pois melhoram sua autoestima e alargam seus grupos de amizades.

Dentre as possibilidades de intervenção universal para o cuidado com a saúde mental na infância e o desenvolvimento socioemocional das crianças, o método FRIENDS, desenvolvido na Austrália pela professora Paula Barrett, tem sido visto nos últimos anos como uma intervenção bem sucedida (Liddle \& Macmillan, 2010). O FRIENDS, como caracterizam Kösters et al. (2012), é um método que ensina habilidades comportamentais para as crianças lidarem, de modo mais eficaz, com os sentimentos de ansiedade e depressão e para construir resiliência emocional, capacidade de resolver problemas e autoconfiança. Os estudos da eficácia desse método foram realizados em vários países, e todos têm mostrado resultados positivos na diminuição dos sintomas de ansiedade, não apenas imediatamente após a conclusão do método, mas também 1 a 3 anos mais tarde (Barrett, Moore, \& Sonderegger, 2000; Barrett, 
Sonderegger, \& Xenos, 2003; Kösters et al., 2012; Mostert \& Loxton, 2008; Stallard, Simpson, Anderson, Hibbert, \& Osborn, 2007).

As letras da sigla FRIENDS, adaptadas para o português como AMIGOS, ajudam a entender os objetivos do método: A refere-se à atenção aos sentimentos (falar sobre seus sentimentos e prestar atenção nos sentimentos dos outros); $\mathbf{M}$ momento de relaxar (técnicas para auto acalmar-se, como de respiração por exemplo); I ideias positivas (aprender a olhar o lado positivo da vida e a lidar com pensamentos que paralisam as ações); G gerar soluções (desenvolver habilidades de resolução de problemas a partir do plano passo a passo); 0 orgulhe-se do seu trabalho (aprender a reconhecer pessoas modelo, como pais, professores, membros da comunidade, que ajudam a torná-lo uma pessoa melhor); e, por fim, o S sorria e lembre-se de ficar calmo (praticar as habilidades aprendidas no método todos os dias com a família e os amigos). Além desses, o método também tem como foco desenvolver hábitos de vida saudável (Barrett, 2012).

Segundo Siu (2007), o método possui três componentes principais: o fisiológico, o cognitivo e o comportamental. As habilidades ensinadas fisiologicamente incluem o aumento da consciência das crianças para os sinais corporais, para que identifiquem quando estão se sentindo preocupadas (taquicardia, por exemplo), aprendendo a respiração profunda e o relaxamento muscular progressivo. Nas habilidades cognitivas, as crianças compreendem que o pensamento afeta o modo como a pessoa se sente e tudo o que ela faz, e com isso são ensinadas a reconhecerem pensamentos que não ajudam, para que os desafiem e acabem com eles, concentrando-se nos aspectos positivos da situação a que estão expostos. Por fim, as competências comportamentais incluem ensinar resoluções de problemas e que elas aprendam a recompensar a si mesmas por terem usado as habilidades de enfrentamento positivo.

Conforme evidenciam Shortt, Barrett e Fox (2001), o método FRIENDS incentiva as crianças a pensarem em seu corpo como seu amigo, pois percebem que ele informa quando estão se sentindo preocupados ou nervosos, a se recompensar quando executam algo difícil, a fazer novas amizades, construir redes sociais e a conversar com alguém quando se encontram em situações que, por algum motivo, os angustiam.

Uma habilidade que se propõe desenvolver no método é a resiliência que, para Pahl e Barrett (2007), é uma habilidade de fundamental importância para a sobrevivência, visto que nenhuma criança é imune à pressão do mundo acelerado e cheio de stress. O método FRIENDS é baseado nessa concepção, de que a maioria das crianças vai sentir pressão em alguma fase da sua vida e é importante fornecer-lhes as habilidades de enfrentamento eficazes e necessárias para "recuperar-se" nessas situações. Enfim, como coloca Stallard (2010), o método FRIENDS, além de ensinar habilidades para a vida como um todo, fornece às crianças uma caixa de ferramentas emocionais para ajudá-las a controlar eficazmente suas emoções.

Inúmeras pesquisas, em mais de 20 países, comprovam a eficácia do FRIENDS (Kösters et al., 2012; Liddle \& Macmillan, 2010; Lowry-Webster, Barrett \& Lock, 2003; Stallard et al., 2007), sendo que o método já foi aprovado pela Organização Mundial da Saúde (Who, 2004). No Brasil, o número de pesquisas acerca do método ainda é bastante limitado, visto que foi implantado recentemente no país (Figueredo, Nico, \& Leonardi, 2015). Nesse sentido, justifica-se a presente pesquisa. Tal método, reconhecido pelo maior órgão estadunidense de análise de programas baseados em evidências científicas National Registry of EvidenceBased Practices (NREPP, 2012), também pode trazer contribuições importantes no cenário 
nacional, uma vez que se sabe que intervenções precoces são bastante efetivas, trazendo tanto benefícios individuais, no que diz respeito ao bem-estar atual e futuro das crianças, como também pode servir a sociedade como um todo, reduzindo os custos sociais relacionados a utilização de cuidados de saúde, medicação e abandono escolar. Assim sendo, essa pesquisa teve como objetivo verificar se o método FRIENDS promoveu melhorias em comportamentos relacionados a capacidades (comportamentos pró-sociais) e dificuldades (hiperatividade, sintomas emocionais, problemas de conduta e problema de relacionamento com os colegas) de dez crianças de uma cidade do interior do Paraná.

\section{Método}

\section{Participantes}

Participaram deste estudo dez crianças de ambos os sexos (03 meninas e 07 meninos), na faixa etária de 6 a 7 anos. A amostra da pesquisa envolveu uma criança que aguardava na fila de espera para atendimento psicológico em uma Clínica Escola de Psicologia de uma Universidade Pública, e nove crianças de uma Escola Privada de um município no interior do Paraná. A seleção da criança da fila de espera se deu em razão de a idade dela coincidir com a faixa-etária delimitada para o grupo. Entrou-se em contato por telefone com os pais, para explicar sobre o grupo e sua oferta como modalidade de atendimento à criança. Diante do aceite, agendou-se a entrevista com os pais. Das outras nove crianças, o primeiro contato aconteceu por meio de divulgação nas escolas, onde foi enviado bilhete para os pais com informações do projeto e o contato da clínica-escola. Para os pais que primeiro entraram em contato com a clínica-escola, as pesquisadoras agendavam a primeira entrevista com os pais e, dado o aceite para participação no grupo, encaminhavam-se os próximos procedimentos para realização destes.

\section{Instrumento}

Strengths and Difficulties Questionnaire (SDQ) - Foi utilizado o questionário de capacidades e dificuldades de Goodman (1997), que detecta problemas relacionados à saúde mental infanto-juvenil de crianças e adolescentes com idades de 3 a 16 anos. O SDQ é composto por 25 itens, sendo 10 itens sobre capacidades, 14 itens sobre dificuldades e um item neutro. $\mathrm{O}$ instrumento é apresentado em três versões, indicadas para serem respondidas pelas próprias crianças (quando estas tiverem mais de 11 anos) ou por seus pais/responsáveis ou ainda pelos professores. No caso desta pesquisa, o questionário foi aplicado para os pais ou responsáveis responderem acerca da criança. Foram participantes dez pais, dentre estes, sete mães, dois pais e um casal, todos com faixas-etárias entre 30 e 40 anos.

O instrumento é divido em cinco subescalas, cada uma com cinco afirmações que visam analisar sintomas emocionais (medos, preocupações excessivas, tristeza e desesperança), problemas de conduta (irritabilidade, agressividade, comportamentos antissociais como mentiras), hiperatividade (inquietação, distração, desatenção), problemas de relacionamento com colegas (dificuldades na relação com outras pessoas, sejam crianças ou adultos) e comportamento pró-social (saber cooperar, ajudar, compartilhar). As alternativas para resposta apresentam como opções falso (zero ponto para esse tipo de resposta), mais ou me- 
nos verdadeiro (um ponto) e verdadeiro (dois pontos), podendo ser assinalada apenas uma única opção por item. Para cada uma das cinco subescalas, a pontuação pode variar de 0 a 10. A interpretação para as escalas de dificuldades será melhor quando o resultado for uma menor pontuação, ao contrário da escala pró-social, em que, quanto maior a pontuação, melhor o resultado.

\section{Método FRIENDS}

A estrutura dos encontros do método FRIENDS está contemplada na tabela a seguir:

Tabela 1

Estrutura dos encontros do método FRIENDS (baseado em Barrett, 2012)

\begin{tabular}{|c|c|}
\hline $\begin{array}{l}\text { Número da } \\
\text { sessão }\end{array}$ & Conteúdo/objetivos \\
\hline 1 & $\begin{array}{l}\text { Introdução: Senso de identidade (quem sou eu, quem você é para os outros); não } \\
\text { há problemas em sentir medo, todos podemos aprender a ser corajosos; todos } \\
\text { temos semelhanças e diferenças. }\end{array}$ \\
\hline 2 & $\begin{array}{l}\text { Meus sentimentos: Identificação de sentimentos em si mesmo e nos outros; } \\
\text { encenação de sentimentos; normalização de todos os sentimentos; aprender a } \\
\text { fazer sentimentos felizes crescerem. }\end{array}$ \\
\hline 3 & $\begin{array}{l}\text { Seus sentimentos: eu vou ajudar: Prestar atenção aos sentimentos de outras } \\
\text { pessoas da família, como os pais, irmãos, avós, professores, amigos; ajudar } \\
\text { outras pessoas a se sentirem melhor (treinamento de empatia); reconhecer os } \\
\text { sentimentos dos outros. }\end{array}$ \\
\hline 4 & $\begin{array}{l}\text { Nossos corpos e jogos de relaxamento: Prestar atenção ao que seu corpo está lhe } \\
\text { dizendo; praticar técnicas de respiração; realizar jogos de relaxamento. }\end{array}$ \\
\hline 5 & $\begin{array}{l}\text { Pensamentos "vermelhos" e "verdes": Pensamentos Vermelhos que não ajudam } \\
\text { - que fazem você parar; pensamentos Verdes que ajudam - que fazem você } \\
\text { continuar! }\end{array}$ \\
\hline 6 & $\begin{array}{l}\text { Transformando pensamentos "vermelhos" em pensamentos "verdes": mudar os } \\
\text { pensamentos "vermelhos" para "verdes"; acolher os pensamentos "vermelhos" } \\
\text { pode ser bem fácil. }\end{array}$ \\
\hline 7 & $\begin{array}{l}\text { Fazer coisas passo a passo: Aprender a fazer as coisas passo a passo; dividir as } \\
\text { coisas difíceis em pequenos passos; aprender a ser corajoso; }\end{array}$ \\
\hline 8 & $\begin{array}{l}\text { Passos para ser um bom amigo: Como ser simpático e fazer novos amigos; ser um } \\
\text { bom amigo: sorrir, compartilhar, ajudar e ouvir. }\end{array}$ \\
\hline 9 & $\begin{array}{l}\text { Fazendo elogios a si mesmo: Como autorrecompensar-se quando tentou o seu } \\
\text { melhor. }\end{array}$ \\
\hline 10 & $\begin{array}{l}\text { Família, escola, vizinhos e amigos: Pessoas modelo e sua influência de exemplo na } \\
\text { vida das crianças; família, amigos e professores podem ajudar a nos tornar mais } \\
\text { corajosos e podemos ajudá-los também. }\end{array}$ \\
\hline 11 & Retomada dos temas desenvolvidos ao longo dos encontros. \\
\hline 12 & Retomada dos temas desenvolvidos ao longo dos encontros e Follow up \\
\hline
\end{tabular}

Além dos conteúdos específicos abordados, realizou-se também, em todos os encontros, o treino de habilidades transversais, em que se buscava ensinar as crianças a: 1) discriminar pequenos momentos felizes que acontecem no dia a dia; 2) aprender a ser corajoso, ou seja, experimentar situações novas, conversar com as pessoas sorrindo e olhando nos 
olhos; 3) desenvolver também o exercício de aprender a ser grato pelas pequenas coisas que acontecem e 4) aprimorar hábitos de vida saudável, como cuidados com a alimentação, importância de tomar água, dormir bem e praticar exercícios ao ar livre.

Aconteceram também três encontros com os pais, os quais envolveram temáticas como: 1) apresentação do método; 2) objetivos a serem alcançados com a participação nele; 3) explicação de cada temática dos encontros, enfatizando de que maneira os pais poderiam praticá-las em casa, deixando claro que, por serem habilidades que estavam sendo desenvolvidas, necessitavam de treinos diários; 4) discussão sobre hábitos de vida saudável, como alimentação, exercícios físicos e rotina de sono.

\section{Procedimento de coleta de dados da pesquisa}

Primeiramente realizou-se o contato com uma Clínica-Escola de Psicologia de uma Universidade Pública no interior do Paraná a fim de conseguir a autorização para a realização da pesquisa no local. Posterior ao consentimento e assinatura do termo de anuência, o projeto de pesquisa foi submetido para avaliação pelo Comitê de Ética em Pesquisa (COMEP), sendo aprovado CAAE 55858916.1.0000.0106. Após a aprovação pelo COMEP, o projeto foi divulgado para as crianças e seus responsáveis, tanto aquelas que estavam no cadastro da fila de espera para atendimento psicológico na Clínica-Escola, quanto em uma escola pública e uma privada. Os interessados deixaram seus contatos na secretaria da clínica.

Foi realizado o contato inicialmente com os responsáveis, individualmente, para a explicação da pesquisa, dos objetivos e obtenção de assinatura no Termo de Consentimento Livre e Esclarecido (TCLE). Dado o consentimento dos responsáveis, foi-lhes solicitado que respondessem ao instrumento SDQ, que foi aplicado individualmente pela pesquisadora.

Antes de iniciar o primeiro encontro do método FRIENDS, foram apresentados às crianças participantes da pesquisa os objetivos desta bem como o Termo de Assentimento do Menor, em linguagem adequada para a faixa etária. Após o aceite, deu-se início ao primeiro encontro. Logo após a finalização dos dez encontros, foram coletados dados novamente com o SDQ. Por fim, após dois meses da participação no método, foi realizada nova coleta de dados com o mesmo instrumento, caracterizando-se como uma medida de follow-up. A coleta de dados deu-se durante todo o processo com os pais, não sendo coletados dados com as crianças em si.

Os encontros contaram com a presença de sete alunas do curso de Psicologia. No grupo das crianças, duas eram facilitadoras e tinham como função explicar sobre os conteúdos de cada encontro e de cada atividade e conduzir a intervenção. Três eram co-facilitadoras que auxiliavam o andamento das atividades, oferecendo suporte para o que as facilitadoras e/ou crianças necessitassem ao longo do encontro, e ajudando também no controle de possíveis comportamentos que pudessem dificultar o andamento das atividades. Os encontros com os pais eram conduzidos por outras duas alunas, explicando-se a necessidade de treinos diários das habilidades que estavam sendo desenvolvidas.

\section{Análise de dados}

Foi realizada a análise de dados a partir do teste não paramétrico Wilcoxon (Wilcoxon, 1945), que se baseia na comparação de duas amostras pareadas. O objetivo desse teste 
consiste em comparar as performances de cada sujeito no sentido de verificar se existem diferenças significativas entre os seus resultados em duas situações. Visto que os dados coletados nessa pesquisa consistiram em pré-teste, pós-teste e follow up, foi possível comparar características relacionadas a capacidades e dificuldades dos participantes antes, depois e dois meses após a participação no método FRIENDS, de acordo com a visão dos pais.

\section{Resultados}

Antes da apresentação dos resultados, é importante salientar que os dados apresentados, referentes à amostra da pesquisa, variaram no follow-up. No procedimento de pré e pós-teste, os responsáveis por dez participantes, número das crianças que iniciou o método, preencheram o instrumento. Já no follow-up, a amostra foi de oito participantes, devido ao fato de os pais de duas crianças não responderem ao SDQ nessa etapa. A análise das amostras com número de participantes diferentes foi possível porque o teste Wilcoxon calcula a diferença entre a primeira e a segunda medida do mesmo par, excluindo os resultados igual a zero, portanto, quando os resultados de pré e pós-teste foram comparados com o follow up, consideraram-se oito participantes, enquanto o pareamento entre pré e pós-teste comparou os dez.

Os resultados referentes à média e desvio padrão das escalas do SDQ foram obtidos, sendo apresentados na Tabela 2.

Tabela 2

Resultados da média e desvio-padrão dos participantes ao instrumento SDQ

\begin{tabular}{l|c|c|c|c|c|c}
\hline \multirow{2}{*}{ Escalas do SDQ } & \multicolumn{2}{|c|}{ Pré-teste } & \multicolumn{2}{c|}{ Pós-teste } & \multicolumn{2}{c}{ Follow-up* } \\
\cline { 2 - 8 } & Média & $\begin{array}{c}\text { Desvio- } \\
\text { padrão }\end{array}$ & Média & $\begin{array}{c}\text { Desvio- } \\
\text { padrão }\end{array}$ & Média & $\begin{array}{l}\text { Desvio- } \\
\text { padrão }\end{array}$ \\
\hline Comportamento pró-social & 9,00 & 1,054 & 9,20 & 1,317 & 9,00 & 1,309 \\
\hline Hiperatividade & 4,80 & 2,700 & 2,30 & 1,636 & 3,50 & 2,000 \\
\hline Sintomas Emocionais & 5,40 & 2,319 & 2,10 & 1,317 & 2,00 & 0,926 \\
\hline Problemas de Relacionamento com colegas & 1,40 & 1,430 & 0,90 & 0,876 & 1,38 & 0,916 \\
\hline Problemas de Conduta & 2,10 & 2,331 & 0,70 & 0,823 & 0,88 & 1,126 \\
\hline TOTAL- Somatória de dificuldades & 13,70 & 7,424 & 6,00 & 2,357 & 7,63 & 4,033 \\
\hline
\end{tabular}

*8 participantes

A partir das comparações das médias, foi possível perceber que todos os escores referentes à escala negativa, isto é, comportamentos inadequados, os quais quanto menor for a média melhor é o resultado, apresentaram diminuição após a participação no método. Isso demostra que o FRIENDS foi eficaz na mudança comportamental das crianças.

Referente ao comportamento pró-social, item da escala positiva, na qual quanto maior a média melhor o resultado, percebe-se que as crianças já apresentavam altos escores e que esse resultado se manteve nas três etapas.

A Tabela 3 apresenta os resultados do teste Wilcoxon. Estão destacados aqueles valores que se mostraram estatisticamente significativos, isto é, os que apontaram resultado de $p<0,05$. 
Tabela 3

Comparação dos três momentos de avaliação a partir do teste Wilcoxon

\begin{tabular}{l|c|c|c|c|c|c}
\hline \multirow{2}{*}{ Escalas do SDQ } & \multicolumn{2}{|c|}{ Pré-teste/Pós-teste } & \multicolumn{2}{c|}{ Pré-teste/Follow-up } & \multicolumn{2}{c}{ Pós-teste/Follow-up } \\
\cline { 2 - 7 } & $\mathrm{Z}$ & $\mathrm{P}$ & $\mathrm{Z}$ & $\mathrm{P}$ & $\mathrm{Z}$ & $\mathrm{P}$ \\
\hline Pró-social & $-0,707$ & 0,480 & $-1,000$ & 0,317 & 0,000 & 1,000 \\
\hline Hiperatividade & $-2,692$ & 0,007 & $-1,732$ & 0,083 & $-2,232$ & 0,026 \\
\hline Sintomas Emocionais & $-2,534$ & 0,011 & $-2,316$ & 0,021 & $-0,520$ & 0,603 \\
\hline $\begin{array}{l}\text { Problemas de Relacionamento } \\
\text { com colegas }\end{array}$ & $-1,089$ & 0,276 & $-0,577$ & 0,564 & $-0,756$ & 0,450 \\
\hline Problemas de Conduta & $-1,980$ & 0,048 & $-0,378$ & 0,705 & $-1,732$ & 0,083 \\
\hline $\begin{array}{l}\text { TOTAL - Somatória de } \\
\text { dificuldades }\end{array}$ & $-2,807$ & 0,005 & $-1,975$ & 0,48 & $-1,845$ & 0,065 \\
\hline
\end{tabular}

É possível perceber que, no comparativo entre pré e pós teste, os resultados estatisticamente significativos foram os da hiperatividade, sintomas emocionais, problemas de conduta e a somatória das dificuldades. Dentre as médias apresentadas entre pré-teste e follow-up, o resultado estatisticamente significativo apresentado foi o de sintomas emocionais, enquanto que, no pós teste e follow-up, esse resultado apresentou-se na hiperatividade. Ou seja, quando comparadas as médias de antes do método com a de dois meses após seu término, as crianças apresentaram diferenças nos sintomas emocionais, assim como a comparação entre a finalização dos encontros com dois meses mais tarde, elas apresentaram diferenças nos sintomas de hiperatividade. Esses escores demonstram que os resultados obtidos com o FRIENDS continuam acontecendo mesmo após seu encerramento.

\section{Discussão}

Foi possível perceber, a partir dos escores apresentados nas Tabelas 2 e 3, que a participação das crianças no método FRIENDS acarretou diminuições em dificuldades relacionadas a hiperatividade, sintomas emocionais e problemas de conduta, demonstrando a efetividade do método tanto imediatamente após a participação quanto no follow-up dois meses mais tarde.

É interessante salientar que as médias e os resultados estatisticamente significativos $(p<0,05)$ de dificuldades apresentados pela amostra não dizem respeito a comportamentos relacionados à interação social, visto que itens como problemas de relacionamento com os colegas indicaram uma média baixa, e o comportamento pró-social apontou resultados elevados, ambos não demonstrando diferenças expressivas quando comparados entre as três medidas. Isto faz com que se levante a hipótese de que esses dados não se demonstram significativos devido ao fato de as crianças já apresentarem repertório comportamental de interação social adequado antes do método, e que isso se manteve.

Quanto aos outros itens referentes à hiperatividade, sintomas emocionais, problemas de conduta e somatório de dificuldades, todos com médias mais elevadas ao início do programa, observaram-se resultados significativos na comparação com pós-teste e follow-up; demonstram que a participação no método contribuiu para diminuição de dificuldades das crianças, no que diz respeito a comportamentos relacionados a agitação, desatenção, agressividade, irritabilidade, ansiedade e características depressivas, por exemplo. Tais dados confirmam que 
- FRIENDS auxilia a lidar mais eficazmente com os sintomas internalizantes e externalizantes, constrói resiliência emocional e capacidade de resolver problemas (Kösters et al., 2012).

Especificamente relacionado às diferenças apresentadas na hiperatividade, a diminuição nos índices após a participação no método podem ser decorrência do aumento da consciência das crianças para os sinais somáticos que indicam que estão ficando mais agitadas ou nervosas, por terem aprendido habilidades de autoacalmar-se a partir da respiração e relaxamento muscular progressivo, o que possibilita a autorregulação, e por aprenderem que o pensamento afeta o modo como a pessoa se sente e o que faz (Siu, 2007). Além disso, o fato de terem aprendido técnicas de enfrentamento como o "ser corajoso" e o "plano passo a passo" isto é, enfrentar as situações e realizá-las em pequenas etapas da mais fácil até chegar a mais complexa, pode ter feito com que as crianças passassem a encarar situações de maneira mais assertiva, ficando menos agitadas e mais atentas na realização das tarefas (Barret, Sondergger, \& Xenos, 2003; Koster et al., 2012; Lidlle \& Maccmillan, 2010; Mostert \& Loxton, 2008).

Referente às melhorias apontadas nos sintomas emocionais, o método também demonstrou cumprir com o objetivo do aumento da competência socioemocional das crianças, ensinando estratégias de regulação de emoções, controle do estresse, desenvolvimento de habilidades que propiciem o alargamento dos grupos de amizade e relacionamentos com pares e adultos que possibilitam uma rede de apoio, promoção de resiliência e superação de adversidades e validação de sentimentos em si e no outro (Dohl, 2008; Pahl \& Barrett, 2007; Stallard, 2010; Stallard et al., 2005). O desenvolvimento de tais habilidades é muito importante, pois como destaca Dohl (2008), crianças que não desenvolvem habilidades socioemocionais não conseguirão realizar plenamente seu potencial, seja ele pessoal, acadêmico ou social e, além disso, a falta de um desenvolvimento emocional saudável está significativamente atrelada ao risco de doença mental.

Aos problemas de conduta estão relacionados os comportamentos não aceitos socialmente, como é o caso da agressividade, birras e não obediência. De acordo com Poulou (2005), crianças que desenvolvem competências emocionais e sociais são menos propensas a apresentar comportamentos violentos e agressivos. Como os dados mostraram que houve diferenças significativas no que diz respeito aos problemas de conduta, infere-se que o método FRIENDS foi eficiente também na diminuição desses comportamentos, por meio do desenvolvimento de habilidades e competências socioemocionais que contribuem para autorregulação emocional, bem como desenvolvimento de repertórios de solução de problemas (Pahl \& Barret, 2007).

De forma geral, tais resultados obtidos na pesquisa mostraram diminuições significativas nas dificuldades das crianças que participaram do método, sobretudo no que diz respeito a hiperatividade, sintomas emocionais e problemas de conduta, demostrando que o FRIENDS foi eficaz para a melhora de tais comportamentos das crianças de uma cidade do interior do Paraná. Isso evidencia que o Método FRIENDS é uma alternativa válida para ser implantada como um programa de prevenção e promoção de saúde, visando a uma atuação antes que problemas mentais apareçam na vida de crianças ou até mesmo atuando para que se evite o agravamento dessas doenças na população, podendo ser aplicado tanto como intervenção universal, selecionada ou indicada.

Cabe ainda destacar as dificuldades deste estudo, tendo em vista possíveis aplicações futuras do método. Destaca-se a longa distância da clínica-escola do centro da cidade, 
dificultando o acesso dos participantes, e a baixa adesão a projetos preventivos, em especial, no follow up. Uma alternativa para essas dificuldades está relacionada à possibilidade de realizar o FRIENDS em escolas e CRAS, como forma de aproximar o método da população. A eficácia do Método FRIENDS foi avaliada e comprovada em vários países (Barrett, Sonderegger \& Xenos, 2003; Kösters et al., 2012; Liddle \& Macmillan, 2010; Stallard et al., 2007), no entanto é uma área de estudos bastante nova no Brasil, sendo esta uma das primeiras pesquisas realizadas. Tanto o fato de esta pesquisa se configurar como umas das pioneiras no cenário nacional, quanto por delimitar um recorte específico de faixa-etária e região da amostra, demonstra-se a necessidade de mais estudos que avaliem sua eficácia no país. Além disso, visto o alto índice de crianças com acometimentos na saúde mental e a carência de implantações de programas de intervenção com foco na prevenção e promoção de saúde, sugere-se a aplicação do método FRIENDS como um meio de prevenção e até minimização desses índices. Nesse sentido, conclui-se que mais estudos nacionais são necessários, em diversos outros contextos e com outras faixas-etárias, visando a dados mais robustos para a confirmação da eficácia do método FRIENDS no Brasil. Além disso, dada a necessidade de implantações de programas que busquem prevenção e promoção de saúde, o método FRIENDS apresenta-se como uma alternativa plausível para diversas populações.

\section{Agradecimentos}

Agradecemos ao Instituto Brasileiro de Inteligência Emocional e Social (IBIES) pela parceria que possibilitou a realização deste estudo.

\section{Referências}

Abreu, S., Barletta, J. B., \& Murta, S. G. (2015). Prevenção e promoção de saúde mental: Pressupostos teóricos e marcos conceituais. In S. G. Murta, C. Leandro-Grança, K. B. Santos, L. Polejack (Eds.), Prevenção e promoção em saúde mental: Fundamentos, planejamento e estratégias de prevenção. São Paulo: Sinopsys.

Barrett, P. M. (2012). Amigos Divertidos: Guia do Facilitador para construção de resiliência em crianças de 4 a 7 anos através do brincar (3a ed.). Austrália: Barrett Research Resources PTy Ltd.

Barrett, P. M.; Moore, A. F.; \& Sonderegger, R. (2000). The FRIENDS program for Young formerYugoslavian refugees in Australia: A pilot study. Behaviour Change, 17, 12-21.

Barrett, P. M., Sondergger, R., \& Xenos, S. (2003). Using FRIENDS to combat anxiety and adjustment problems among young migrants to Australia: A national trial. Clinical Child Psychology and Psychiatry, 8(2), 241-260.

Barros, M. G. S. M. (2005). Estudo de prevalência de problemas de saúde mental em adolescentes de 11 a 16 anos na cidade de Barretos (Dissertação de Mestrado, Universidade Presbiteriana Mackenzie, São Paulo).

Dohl, A. H. (2008) Managing anxiety through childhood social-emotional development (Mestrado em Artes, Universidade da Colúmbia Britânica).

Figueredo, U. S. P.; Nico, Y.; \& Leonardi, J. L. (2015) A experiência do Método Friends: uma possibilidade de prevenção e promoção de saúde mental em larga escala no Brasil. Boletim Paradigma $10,20-24$. 
Fleitlich-Bil, Y. K. B., \& Goodman, R. (2004). Prevalence of child and adolescent psychiatric disorders in southeast Brazil. Journal of American Academy of Child and Adolescent Psychiatry, 43(6), 727-734.

Fraga, B. P. (2015) Depressão Infantil: Uma revisão da literatura. Universidade Federal do Rio Grande do Sul (Especialização em Infância e Família: Avaliação, Prevenção e Intervenção, Instituto de Psicologia, Porto Alegre).

Goodman R. (1997). The Strengths and Difficulties Questionnaire: A research note. Journal of Child Psychology and Psychiatry, 38(5), 581-586.

Goodman, R.; \& Scott, S. (2012). Child and adolescente psycuiatry (3a ed.). Oxford, UK: Wiley-Blackwell.

Kösters, M. P.; Chinapaw, M. J.; Zwaanswijk, M.; Van Der Wal, M. F.; Mwj Utens, E.; \& Koot, H. M. (2012). Study design of 'FRIENDS for life': Process and effect evaluation of an indicated school-based prevention programme for childhood anxiety and depression. Bmc public health, 12(86).

Liddle, I., \& Macmillan. S. (2010). Evaluating the FRIENDS programme in a Scottish setting. Educational Psychology in Practice, 26(1), 53-67.

Lowry-Webster H. M.; Barrett, P. M.; \& Lock. S. (2003) A universal prevention trial of anxiety symptomology during childhood: results at 1-year follow-up. Behav Chang, 20(1), 25-43.

Maia, J. M. D., \& Williams, L. C. A. (2005). Fatores de risco e fatores de proteção ao desenvolvimento infantil: uma revisão da área. Temas em Psicologia, 13(2), 91-103.

Martin-Raugh, M. P.; Kell, H. J.; \& Motowidlo, S. J. (2016). Prosocial knowledge mediates effects of agreeableness and emotional intelligence on prosocial behavior. Personality and Individual Differences, 90, 41-49.

Miller, L. D., Laye-Gindhu, A., Liu, Y; March, J. S., Thordarson, D. S., \& Garland, E. J. (2011). Evaluation of a preventive intervention for child anxiety in two randomized attentioncontrol school trials. Behaviour Research and Therapy, 49(5), 315-23.

Mostert, J., \& Loxton, H. (2008). Exploring the Effectiveness of the FRIENDS Program in Reducing Anxiety Symptoms Among South African Children. Behaviour Change, 25(2), 85-96.

Murta, S. G., Günther, I. A., \& Guzzo, R. S. (2015). Prevenção e promoção em saúde mental no curso de vida: indicadores para a ação. In S. G. Murta, C. Leandro-Grança, K. Santos B., \& L. Polejack. Prevenção e promoção em saúde mental: Fundamentos, planejamento e estratégias de prevenção (pp. 75-92). São Paulo: Sinopsys editora.

Muszkat, M., Miranda, \& Mc., Rizutti, S. (2011). Transtorno do déficit de atenção e hiperatividade. São Paulo: Cortez.

National Registration of evidence-based programs and pratices. (2012). NREPP.

Pahl, K. M.; \& Barrett, P. M. (2007). The Development of Social-Emotional Competence in Preschool-Aged Children: An Introduction to the Fun FRIENDS Program. Australian Journal of Guidance \& Counselling, 17(1), 81-90.

Poulou, M. (2005). The prevention of emotional and behavioural difficulties in schools: Teachers' suggestions. Educational Psychology in Practice, 21(1), 37-52.

Saud, L. F.; \& Tonelotto, J. M. F. (2005). Comportamento social na escola: diferenças entre gêneros e séries. Psicol. esc. educ., 9(1), 47-57. 
Siu, A. F.Y. (2007). Using FRIENDS to combat internalizing problems among primary school children in Hong Kong. Journal of Cognitive and Behavioral Psychotherapies, 7(1), 11-26.

Shortt, A. L.; Barrett, P.M.; \& Fox, T. L. (2001). Evaluating the FRIENDS Program: A CognitiveBehavioral Group Treatment for Anxious Children and Their Parents. Journal of Clinical Child \& Adolescent Psychology, 30(4), 525-535.

Stallard, P. (2010). Mental health prevention in UK classrooms: The FRIENDS anxiety prevention programme. Emotional and Behavioural Difficulties, 15(1), 23-35.

Stallard, P.; Simpson, N; Anderson, S; Carter, T; Osborn, C; \& Bush, S. (2005). An evaluation of the FRIENDS programme: a cognitive behaviour therapy intervention to promote emotional resilience. Arch. Dis. Child., 90(10), 1016-1019.

Stallard, P.; Simpson, N.; Anderson, S.; Hibbert, S.; \& Osborn, C. (2007). The FRIENDS emotional health programme: Initial findings from a school-based project. Child and Adolescent Mental Health, 12(1), 32.

Stallard, P.; Simpson, N.; Anderson, S.; \& Goddard, M. (2008). The FRIENDS emotional health prevention Programme. 12 month follow-up of a universal UK school based trial, Eur Child Adolesc Psychiatry, 17, 283-289.

Stallard, P.; Skryabina, E.; Taylor, G.; Phillips, R.; Daniels, H.; Anderson, R.; \& Simpson, N. (2014). Classroom-based cognitive behaviour therapy (FRIENDS): a cluster randomised controlled trial to Prevent Anxiety in Children through Education in Schools (PACES), Lancet Psychiatry, 1(3), 185-92.

Teste de Wilcoxon. (1945). Disponível em http://www.inf.ufsc.br/ vera.carmo/Testes_de_ Hi\%20poteses/Teste_nao_parametrico_Wilcoxon.pdf

World Health Organization. (2004). Prevention of mental disorders: effective interventions and policy options: summary report/a report of the World Health Organization Dept. of Mental Health and Substance Abuse; in Collaboration with the Prevention Research Centre of the Universities of Nijmegen and Maastricht. WHO.

Recebido: $12 / 09 / 2017$

Última revisão: 18/12/2017

Aceite final: 15/08/2018

\section{Sobre os autores:}

Cynthia Lais Ignachewski - Acadêmica do 5o ano do curso de Psicologia da Universidade Estadual do Centro-Oeste. E-mail: cyn_lais@windowslive.com, Orcid: http://orcid.org/0000-0001-7050-785X

Ana Priscila Batista - Professora Doutora do Departamento de Psicologia da Universidade Estadual do Centro-Oeste. E-mail: anapribatista@yahoo.com.br, Orcid: http://orcid.org/0000-0001-9849-3998

Caroline Guisantes de Salvo Toni - Professora Doutora do Departamento de Psicologia da Universidade Estadual do Centro-Oeste. E-mail: carolineguisantes@yahoo.com.br, Orcid: http://orcid.org/0000-0003-2114-1964

Giulia Tatiana Tkaczyk Pavoski-Acadêmica do 5 o ano do curso de Psicologia da Universidade Estadual do Centro-Oeste. E-mail: gt.pavoski@yahoo.com.br, Orcid: http://orcid.org/0000-0001-9375-4271 
